\title{
Scanning gate imaging of quantum dots in 1D ultra- thin InAs/InP nanowires
}

\section{Citation}

Boyd, Erin E, Kristian Storm, Lars Samuelson, and Robert M Westervelt. 2011. "Scanning Gate Imaging of Quantum Dots in 1D Ultra-Thin InAs/InP Nanowires." Nanotechnology 22 (18) (March 22): 185201. doi:10.1088/0957-4484/22/18/185201.

\section{Published Version}

10.1088/0957-4484/22/18/185201

\section{Permanent link}

http://nrs.harvard.edu/urn-3:HUL.InstRepos:33373341

\section{Terms of Use}

This article was downloaded from Harvard University's DASH repository, and is made available under the terms and conditions applicable to Open Access Policy Articles, as set forth at http:// nrs.harvard.edu/urn-3:HUL.InstRepos:dash.current.terms-of-use\#OAP

\section{Share Your Story}

The Harvard community has made this article openly available.

Please share how this access benefits you. Submit a story.

Accessibility 


\title{
Scanning gate imaging of quantum dots in 1D ultra-thin
}

\section{InAs/InP nanowires}

Erin E Boyd ${ }^{1}$, Kristian Storm $^{2}$, Lars Samuelson ${ }^{2}$ and Robert M Westervelt ${ }^{1,3}$

${ }^{1}$ Department of Physics, Harvard University, Cambridge, MA 02138, USA

${ }^{2}$ Solid State Physics/the Nanometer Structure Consortium, Lund University, Box 118, S-221 00

Lund, Sweden

${ }^{3}$ School of Engineering and Applied Science, Harvard University, Cambridge, MA 02138, USA

Email: westervelt@seas.harvard.edu

\begin{abstract}
We use a scanning gate microscope (SGM) to characterize one-dimensional ultra-thin (diameter $\approx 30 \mathrm{~nm}$ ) InAs/InP heterostructure nanowires containing a nominally $300 \mathrm{~nm}$ long InAs quantum dot defined by two InP tunnel barriers. Measurements of Coulomb blockade conductance $v$ s. backgate voltage with no tip present are difficult to decipher. Using the SGM tip as a charged movable gate, we are able to identify three quantum dots along the nanowire: the grown-in quantum dot and an additional quantum dot near each metal lead. The SGM conductance images are used to disentangle information about individual quantum dots and then to characterize each quantum dot using spatially resolved energy-level spectroscopy.
\end{abstract}




\section{Introduction}

One-dimensional (1D) semiconductor nanostructures, such as nanowires [1-8], carbon nanotubes [9-12], and cleaved-edge overgrowth wires [13-16], offer the opportunity to investigate interesting electronic phenomena in 1D. Select quantum mechanical effects that are not present or vastly more complicated in higher dimensional systems can be explored. Examples of effects that could be studied in 1D systems include the longitudinal electronic wavefunction [17], spincharge separation of charge carriers [15], and transition from a Luttinger Liquid to a Wigner Crystal state $[12,18,19]$. For all of these materials, a very high quality sample with little disorder is needed to create a system where $1 \mathrm{D}$ physics can be accessed and studied. With a 1D sample of sufficiently high quality, the amplitude of the electronic wavefunction along the length of the wire could potentially be measured [20].

InAs/InP heterostructure nanowires are a particularly favorable system to probe 1D semiconductor physics. InAs is known to have a charge accumulation layer at the surface [21], which makes it possible to grow quantum dots in ultra-thin nanowires that are not depleted of electrons. InAs has a large g-factor, which can be varied from the bulk value of $|g|=14$ to 2 depending on the dot size [22], and a small effective mass, bulk value $0.022 m_{e}$, increasing the ease of spin manipulation.

There is great interest in developing very clean, high quality 1D nanowire systems. In order for the nanowires to be quantum mechanically $1 \mathrm{D}$ only one subband can be occupied for moderate electron number at liquid He temperatures. This requires the nanowires to be ultra-thin with a diameter $d_{N W} \sim 20$ to $30 \mathrm{~nm}$. An electron in a nanowire can be approximated as a particle in a cylindrical box with infinite potential walls. The Hamiltonian and electronic wavefunction can be separated into a transverse and a longitudinal piece. By placing the Fermi level below the 
first excited transverse state, only the first subband is occupied and the nanowire is effectively 1D. For a $300 \mathrm{~nm}$ long cylindrical box with a $30 \mathrm{~nm}$ diameter, the bottom of the first subband is at energy $\sim 38 \mathrm{meV}$ (the transverse ground state), and the bottom of the second subband is at $\sim 95 \mathrm{meV}$ (the first excited transverse state). This simple, particle-in-a-box model predicts that a one-dimensional electron gas is formed when only the first subband is occupied, for the first 18 electrons added to the dot. A thorough understanding and control of the transport properties, growth, and structure of semiconductor nanowires is essential for their promised potential to be realized as laboratories for $1 \mathrm{D}$ physics.

Liquid-He cooled scanning gate microscopes (SGMs) have been recognized as valuable tools for increasing the understanding of nanoscale systems, such as two-dimensional electron gases [23-28], carbon nanotubes [29], and graphene [30-32]. A SGM provides high spatial resolution images of conductance, which is beyond the capabilities of typical transport measurements alone. Scanning gate microscopy is well suited to probe 1D systems, because it can locate the position of quantum dots using Coulomb blockade conductance rings, and it offers the ability to perform spatially resolved energy-level spectroscopy on individual dots. A conductance image is made by displaying the conductance through the nanowire $v s$. tip position as the conducting tip is raster scanned at a constant height above the surface, as shown in figure 1. In previous SGM-studies, larger diameter wires were used, for which uncontrolled multiple radial modes were always occupied $[33,34]$. Scanning gate microscopy provides information beyond that of optical microscopy or traditional transport measurements to assist in the development of very high quality $1 \mathrm{D}$ devices.

In this paper we characterize a 1D ultra-thin $(d \sim 30 \mathrm{~nm}) \mathrm{InAs} / \mathrm{InP}$ heterostructure nanowire that contains a grown-in epitaxial InAs quantum dot defined by InP tunnelling barriers, using spatially resolved energy-level spectroscopy. A conducting tip in our home-built liquid- 
He-cooled SGM is used to locally gate an InAs/InP nanowire as shown in figure 1. We spatially map the conductance $G v$ s. the tip position $x_{t i p}$ and $y_{t i p}$. Elliptical rings of high conductance, corresponding to Coulomb blockade conductance peaks, are found to be centered on the epitaxially grown InAs quantum dot. From the images, we find the length of the epitaxially grown quantum dot $l_{\text {expitaxial }}$ to be approximately $350 \mathrm{~nm}$, in good agreement with growth specifications. Two additional quantum dots are also apparent in the conductance images. These additional quantum dots were formed near the metal/semiconductor contacts at the ends of the nanowire. By comparing the ring spacings with that of the center dot, we estimate the sizes of the additional dots. We demonstrate that the SGM's movable gate is a valuable tool that can be used to understand the motion of electrons through ultra-thin heterostructure nanowires and to facilitate the development of growth processes to make highly controllable structures.

\section{Experimental details}

The measurement setup is shown in figure 1. InAs nanowires containing an InAs quantum dot defined by InP tunnel barriers were epitaxially grown on InAs $<111>\mathrm{B}$ substrates using chemical beam epitaxy [35]. Size selected gold aerosol particles were chosen as growth catalysts to produce ultra-thin nanowires with $d \sim 30 \mathrm{~nm}$ and length, $\sim 2$ to $3\lceil\mathrm{~m}$. InAs sections and InP barriers are grown by switching gas precursors during growth. An InAs quantum dot with nominal length $300 \mathrm{~nm}$ was formed by two 2-nm-long InP tunnel barriers. A long quantum dot, one of length much longer than its diameter, was chosen to allow us to probe the electron density profile along the nanowire in order to study the $1 \mathrm{D}$ physics of the electron. The $\sim 0.6 \mathrm{eV}$ conduction band offset between InAs and InP forms a potential well with strong electronic confinement. The In growth source used for the InAs quantum dot was triethylindium (TEIn) and the growth source for the InAs nanowire leads was trimethylindium (TMIn) to enhance carbon doping in the nanowire leads. The carbon n-dopes the nanowire leads relative to the dot, allowing us to lower the electron number on the epitaxially grown quantum dot without 
first depleting the nanowire leads. The heterostructure nanowires were transferred to a degenerately doped Si substrate with $100 \mathrm{~nm}$ of thermal oxide. The doped substrate is used as a non-local gate by applying a backgate voltage $V_{b g}$ between the backgate and the nanowire. Nanowires are electrically contacted with $\mathrm{Ni} / \mathrm{Au}$ electrodes defined using electron-beam lithography. The nanowires that exhibit the Coulomb blockade effect at low temperatures have a resistance between 5 to $10 \mathrm{M}\lceil$ at room temperature. The nanowire sample is cooled in a home-built SGM [24] by He exchange gas in thermal contact with a liquid He bath at $T=4.2 \mathrm{~K}$. At liquid He temperatures, when a large enough source-drain bias is applied so the quantum dot is no longer in the Coulomb blockade regime, the resistances of the nanowires are a few $\mathrm{M}\lceil$.

Using conductance images in the Coulomb blockade regime [26, 33, 34], we determine the location and number of quantum dots in the system and obtain spatial information about the energy-level spectrum throughout the nanowire system. The SGM tip is capacitively coupled to the nanowire; no current flows between the tip and the sample. A conductance image is recorded by displaying the nanowire conductance $G$ as the conducting SGM tip is raster scanned at a constant height above the sample. An example of an image of the conductance of a single quantum dot in a thicker InAs/InP nanowire is shown in figure 2. The charge induced on a dot by the conductive tip is

$$
q_{d o t}\left(r_{t i p}, V_{t i p}\right)=C_{t d}\left(r_{t i p}\right) V_{t i p}
$$

where $V_{t i p}$ is the applied voltage between the SGM tip and the nanowire, $r_{t i p}$ is the distance from the tip to the quantum dot, and $C_{t d}\left(r_{t i p}\right)$ is the capacitance between the dot and the tip when separated by $r_{t i p}$. By changing $V_{t i p}$ or $r_{t i p}$, the induced charge $q_{d o t}$ (equation 1) changes and the chemical potential $/$ dot of the quantum dot shifts and changes the conductance $G$ through the nanowire. Figure 2 schematically represents the change in $G$ as a negatively charged tip is scanned above the dot. In figure 2(a) the tip is to the left of the dot's center and the current is 
blocked, because ${ }_{d}$ dot is out of resonance with the leads. When the negatively charged tip is moved closer to the dot, figure $2(\mathrm{~b}), \Gamma_{d o t}$ is shifted into resonance with the source and the drain and current flows. In figure 2(c), the tip moves closer and gates the dot more strongly and $\Gamma$ dot is shifted further upward and conductance is blocked again. Likewise, moving the tip the same distance away, but to the other side of the dot, has the same effect. Figure 2(d) illustrates a line plot of the conductance through the nanowire $v s$. the positions of the tip. In figure 2(e) the 1D scan is translated into a $2 \mathrm{D}$ conductance image taken by raster scanning the tip in a plane above the nanowire [34]. Each concentric ring of high conductance that appears in the images corresponds to a Coulomb blockade peak in the conductance as an electron is added to or removed from the dot and is centered on the location of a quantum dot [26, 33, 34]. For the small dot in figure 2(e) the high conductivity contours are simply a circle.

The shape of a conductance ring is determined by the capacitance $C_{t d}\left(r_{t i p}\right)$ between the tip and the dot, which depends on the distance between the tip and the quantum dot as the tip is scanned. For a point quantum dot, this will produce circular Coulomb blockade conductance rings as in figure $2[26,34]$. However, if the length of the quantum dot is longer than the tip to nanowire distance, the conductance circles will be stretched along the nanowire axis to form ellipses. Each set of concentric conductance rings is centered on the location of an individual quantum dot, which allows the number and location of the quantum dots in the system to be identified. In the case where multiple dots are present along the length of the wire, the conductance rings for each dot will only be visible when they overlap with the rings from the other dots. The conducting tip operates as a movable gate supplying spatial information about the nanowire, such as dot number, location, and energy-level spectrum, using Coulomb blockade [33, $34]$. 


\section{Results and discussion}

Transport measurements of ultra-thin nanowires yielded Coulomb blockade diamonds with complex behaviour as shown in figure 3(a), which plots the current $I_{\text {sd-offset }}\left(\left[\begin{array}{c}\text { sd-offset } \\ =1\end{array}\right.\right.$ $\left.I_{s d} \mid+10 \mathrm{pA}\right) v s$. backgate voltage $V_{b g}$ and source-drain bias $V_{s d}$, where $\Gamma_{s d}$ is the current through the nanowire. The darker areas of figure 3(a) correspond to regions where the current is blocked by the Coulomb blockade. The many overlapping diamonds in the Coulomb blockade plot indicate that multiple quantum dots were formed along the ultra-thin nanowire. The location and origin of these confined electronic states is not apparent from traditional transport measurements alone, making it difficult to understand their source. The magnitude of the current in the Coulomb blockade plot is plotted on a log scale to accentuate the conductance change at small $V_{s d}$. Figure 3(a) shows that the electron gas in the nanowire is effectively $1 \mathrm{D}$. At $V_{b g}=0 \mathrm{~V}$ the quantum dots are empty of electrons as seen by the last Coulomb blockade diamond opening up for low $V_{b g}$. A positive $V_{b g}$ must be applied to pull electrons onto the dot. The first electrons begin to enter the dot at $V_{b g} \approx 0.4 \mathrm{~V}$. For the range of voltages used in this work, the number of electrons on the epitaxially grown dot is approximately 10, placing the nanowire in the $1 \mathrm{D}$ regime.

Using the SGM tip as a movable gate, we show that the Coulomb blockade diamond pattern was created by three individual quantum dots located along the nanowire. Figure 4(a) shows a conductance image for an ultra-thin nanowire $v s$. tip position $x_{t i p}$ and $y_{t i p}$. The presence of three sets of concentric overlapping rings in figure 4(a) indicates that there are three quantum dots in series along the length of the wire. Each set of concentric rings is centered on a quantum dot. The blue line shown in figure 4(b) marks the location of the nanowire, and the two red blocks denote the approximate positions of the two InP tunnel barriers. The shape of the conductance rings for the quantum dots are well fit by a series of ellipses, shown as the white dotted lines in figure 4(b). Each small solid dark blue circle represents the center for a quantum dot located at 
the center of the Coulomb blockade rings. For clarity, figure 4(c) illustrates the nanowire, barriers, center of the quantum dots, and conductance ring fits without the conductance image.

From the shape and spacing of the conductance rings, we determine that the center dot in figure 4 is the epitaxially grown quantum dot, and we find its length $l_{\text {epitaxial }} \approx 350 \mathrm{~nm}$, in good agreement with the growth parameters. The spatial separation of Coulomb blockade rings is $\left\lceil r\left\lceil 1 / \ell_{d o t}\right.\right.$ for a particular dot, because $\left\lceil r\left\lceil 1 / E_{C}\left\lceil 1 / C_{\lceil}\right.\right.\right.$and $C_{\lceil}\left\lceil l_{d o t}\right.$, where $E_{c}$ is the charging energy of the dot, $C_{\Gamma}$ is the total capacitance of the quantum dot, and $l_{d o t}$ is the length of the particular dot [33]. In figure 4, the center quantum dot has the closest spaced conductance rings, indicating that it is the longest dot. The large eccentricity of the conductance rings shows that the aspect ratio length/diameter for the epitaxially grown dot is substantial.

We find the length $l_{\text {epitaxial }}$ of the epitaxial dot by first measuring the minimum spacing $\left\lceil V_{b g}\right.$ between Coulomb blockade peaks, and then putting this value into a theoretical expression for the backgate-to-dot capacitance $C_{b g d}$. The minimum spacing $\left\lceil V_{b g}\right.$ is extracted from figure 3(b), where a trace of $I_{s d-\text { offset }} v s . V_{b g}$ is taken from a line cut of the Coulomb block plot in figure 3(a) at $V_{s d}=5 \mathrm{mV}$ with no tip present. For a set of isolated quantum dots, the longest dot will have the closest spaced Coulomb blockade conductance peaks, because it has the largest $C_{t d}$ and the smallest charging energy. For multiple quantum dots in series, when the conductance through one dot is blocked no current will flow through the entire nanowire [36, 37]. Figure 3(c) plots $\left\lceil V_{b g} v s . \digamma \Gamma\right.$, where $\Gamma V_{b g}$ is the spacing between adjacent Coulomb blockade peaks from figure 3(b), and $\Gamma \Gamma$ is the change in the number of the Coulomb blockade peaks in figure 3(b). In figure 3(c), we identify an edge defined by a well-defined lowest group of spacings between $\left\lceil V_{b g}=0 \mathrm{mV}\right.$ and $\Gamma V_{b g}=8 \mathrm{mV}$. In figure 3(c), the average $\left\lceil V_{b g}=5.5 \mathrm{mV}\right.$ of this group of smallest spacing is indicated by the dashed line. To find $l_{\text {epitaxial }}$ we model the capacitance $C_{b g d}$ 
between the epitaxial dot and the backgate by $C_{b g d}=2 \Gamma \quad{ }_{r}{ }_{r}{ }_{o} l_{d o t} / \ln (2 z / r)$, the capacitance per unit length between an infinite cylinder and a conducting plane [38], where $r=15 \mathrm{~nm}$ is the nanowire radius, $z=100 \mathrm{~nm}$ is the thickness of the insulating $\mathrm{SiO}_{2}$ layer, $\Gamma_{r}=3.9$ is the dielectric constant of $\mathrm{SiO}_{2}$, and $\Gamma{ }_{o}$ is the permittivity of free space. The logarithmic form is valid when $r$ $<<z$. The measured capacitance $C_{b g d}$ between the backgate and the epitaxially grown dot is $C_{b g d}=e /\left[V_{b g}\right.$, where $e$ is the electron charge, giving $C_{b g d} \approx 30 \mathrm{aF}$. Inserting $C_{b g d}$ into the expression we find $l_{\text {epitaxial }} \approx 350 \mathrm{~nm}$. The measured length of the epitaxially grown InAs dot agrees with the nominal length $300 \mathrm{~nm}$ predicted by the growth conditions. Measuring the expected length of the epitaxially grown dot shows that the electronic wavefunction occupies the full length of the quantum dot, for the small applied bias $V_{s d}=2.5 \mathrm{mV}$. We expect that $V_{s d}$ is smaller than the electrons' Fermi energy, which should be a good fraction of the $98 \mathrm{meV}$ energy of the first excited transverse subband

In addition to the epitaxially grown quantum dot, the SGM images reveal two quantum dots, labeled dot 1 and dot 3 , on either side of the epitaxial dot as shown in figure 4 . The Coulomb blockade rings show that the center of the two additional quantum dots occurs between the epitaxially grown dot and the two metal/semiconductor contacts, suggesting that they are created by Schottky barriers at the interfaces. A small $V_{s d}$ bias must be applied to the nanowire to overcome a small potential barrier and for current to flow, as seen in figure 3. A small Schottky barrier $(\sim 1 \mathrm{meV})$ is apparently formed in order to align the chemical potentials of the metal contacts and the semiconductor nanowire. SGM conductance images disentangle the transport information for the three dots by spatially resolving the Coulomb blockade rings for each dot.

The relative sizes of the three dots are determined by comparing the spacing $\Gamma r$ between the rings in a conductance image for a particular dot with fixed $V_{t i p}$ and $V_{b g}$, such as in 
figure 4 [33]. The spacing $/ r$ is inversely proportional to the capacitance $C_{t d}$ between the SGM tip and that quantum dot. The capacitance $C_{t d}$ between the tip and the cylindrical quantum dot is larger when the tip is directly over the dot. The location of the conductance rings follows a trajectory of constant $C_{t d}$, allowing the elliptical conductance rings to occur at tip positions that are directly above a section of the quantum dot. The smaller the dot, the more energy is required to add an electron to the quantum dot and the greater the spacing between Coulomb blockade conductance rings. For the epitaxial dot, $\Gamma r_{\text {epitaxial }} \approx 26 \mathrm{~nm}$ for $V_{\text {tip }}=-0.6 \mathrm{~V}$. We find the length of the additional dots by comparing the ratios of their ring spacing $\Gamma r_{d o t l}, \Gamma r_{\text {dot3 }}$ with $\Gamma r_{\text {epitaxial }}$. We find for dot 1 the length $l_{\text {dotl }} \approx 300 \mathrm{~nm}$, comparable to the distance between the InP barrier and the metal contact. The spacings between the conductance rings of dot 3 are significantly larger, which indicates that dot 3 is the smallest dot. The length $l_{\text {dot } 3} \approx 50 \mathrm{~nm}$ suggests that dot 3 is defined in part by disorder.

We find the ratio $C_{t d} / C_{b g d}$ of the tip-to-dot capacitance $C_{t d}$ to the backgate-to-dot capacitance $C_{b g d}$ by comparing the change in tip voltage $\left\lceil V_{\text {ele-tip }}\right.$ and the change in backgate voltage $\left\lceil V_{\text {ele-bg }}\right.$ needed to add an electron to the epitaxial dot. Figure 5 shows a series of conductance images taken as the tip voltage is made more negative by changing $V_{\text {tip }}=-0.6 \mathrm{~V}$ to $V_{\text {tip }}=-1.0 \mathrm{~V}$. The scan location is chosen to image the epitaxially grown dot. Each conductance ring corresponds to an increase in the number of the electrons on the dot from $\mathrm{N}$ to $\mathrm{N}+1$. The dashed white ring in figure 5(a)-5(e) serves as a reference point of the location of the $N_{o}$ conductance ring at $V_{\text {tip }}=-0.6 \mathrm{~V}$. The $N_{o}$ ring moves outward in figure 5(a)-5(e), because the tip can more easily push electrons off of the dot when $V_{t i p}$ is more negative (see movie in the online supplemental data). By tracking the progression of the $N_{o}$ conductance ring (the solid white ring in figure 5(a)-5(e)), the distance that the $N_{o}$ ring moves outward from its reference location in figure 5(a) $\left\lceil S\right.$ vs. $V_{\text {tip }}$ is plotted and found to be nearly a straight line indicating that 
$C_{t d}\lceil 1 /\lceil r$ on the length scales of these scans. Using the data shown here, we find $\left\lceil V_{\text {ele-tip }} \approx 0.23 \mathrm{~V}\right.$ is needed to add an electron to the epitaxial dot without moving the tip, when $r_{t i p} \approx 200 \mathrm{~nm}$ from the dot, by sweeping $V_{t i p}$ until the $N_{o}$ conductance ring moves outward by one ring spacing $\Gamma r_{\text {epitaxial }}$.

The backgate voltage change $\left\lceil V_{\text {ele-bg }}\right.$ needed to add an electron is found from figure 6 , which shows a series of conductance images recorded with a fixed $V_{t i p}$ as the backgate voltage $V_{b g}$ is varied from $0.722 \mathrm{~V}$ to $0.714 \mathrm{~V}$. The solid white ring tracks the motion of one conductance ring as $V_{b g}$ is decreased. We use a procedure analogous to that above to find the change $\left\lceil V_{\text {ele-bg }} \approx 0.08 \mathrm{~V}\right.$ needed to add an electron to the dot.

Using data from figures 5 and 6 , we find the tip-to-dot capacitance $C_{t d}$ for the epitaxially grown dot. The ratio $C_{t d} / C_{b g d}$ of the tip-to-dot capacitance and the backgate-to-dot capacitance is found from:

$$
e\left\lceilC _ { t d } \left\lceilV _ { t i p } \left\lceilC _ { b g d } \left\lceil V_{b g}\right.\right.\right.\right.
$$

The capacitance $C_{\text {bgd }}$ between the backgate and the epitaxially grown dot is found from $C_{b g d}=e /\left\lceil V_{b g}\right.$ using $\left\lceil V_{b g}=5.5 \mathrm{mV}\right.$ from figure 3(c). We find $C_{t d} \sim 10 \mathrm{aF}$ when $r_{t i p} \approx 200 \mathrm{~nm}$ from the epitaxially grown dot. This value is much smaller than $C_{\digamma}\lceil\lceil$ showing the tip is effective as a weakly coupled probe of the system.

\section{Conclusion}

We have identified and characterized individual quantum dots in series along an ultra-thin heterostructure nanowire. Without a SGM the location of these dots and their characteristics would have been difficult to discern. We have shown that a well-defined long ultra-thin dot is formed by thin InP tunnel barriers. Evenly spaced concentric elliptical conductance rings are 
centered on the epitaxially grown quantum dot proving that the dot is long and has a large aspect ratio. The length of the dot, calculated by the knowledge of the number of dots in the wire from SGM measurements, and the Coulomb blockade peaks from the transport data, was $l_{\text {epitaxial }} \approx 350 \mathrm{~nm}$, which is within the range expected from the growth. Furthermore, we found two additional dots on either side of the grown-in dot at the metal/semiconductor interfaces and characterized their lengths. In order to add or remove one electron from the epitaxially grown quantum dot a change of $\left\lceil V_{\text {ele-tip }} \approx 0.23 \mathrm{~V}\right.$ or $\left\lceil V_{\text {ele-bg }} \approx 0.08 \mathrm{~V}\right.$ is required, determining the tip-to-dot capacitance $C_{t d} \sim 10 \mathrm{aF}$. With this information, we can now optimize the growth process to reduce the Schottky barriers and control the electron number to produce a simplified system conducive to imaging and probing 1D physical phenomena such as the electronic wavefunction. Through this research we expect to produce high quality ultra-thin heterostructure nanowires that are well suited to understanding a 1D electron gas.

\section{Acknowledgments}

We thank J. Berezovsky, H. Trodahl, and K. Brown for helpful discussions. This research was supported at Harvard University by the NSF funded Nanoscale Science and Engineering Center (NSEC) under grant NSF/PHY06-46094; work at Lund University was supported by the Swedish Research Council (VR), the Swedish Foundation for Strategic Research (SSF) and the Knut and Alice Wallenberg Foundation (KAW).

\section{References}

[1] Wagner R S 1970 Whisker Technology, ed A P Levitt (New York: Wiley) pp 47-119

[2] Hiruma K, Yazawa M, Haraguchi K, Ogawa K, Katsuyama T, Koguchi M and Kakibayashi 1993 GaAs free-standing quantum-size wires J. Appl. Phys. 743162 
[3] Hiruma K, Murakoshi H, Yazawa M and Katsuyama T 1996 Self-organized growth of GaAs/InAs heterostructure nanocylinders by organometallic vapor phase epitaxy J. Crystal Growth 163226

[4] Ohlsson B J, Björk M T, Magnusson H, Deppert K, Samuelson L and Wallenberg L R 2001 Size-, shape-, and position-controlled GaAs nano-whiskers Appl. Phys. Lett. 793335

[5] Björk M T, Ohlsson B J, Sass T, Persson A I, Thelander C, Magnusson M H, Deppert K, Wallenberg L R and Samuelson L 2002 One-dimensional heterostructures in semiconductor nanowhiskers Appl. Phys. Lett. 801058

[6] Björk M T, Ohlsson B J, Sass T, Persson A I, Thelander C, Magnusson M H, Deppert K, Wallenberg L R and Samuelson L 2002 One-dimensional steeplechase for electrons realized Nano Lett. 287

[7] Lieber C M 2003 Nanoscale science and technology: building a big future from small things MRS Bull. 28486

[8] Fuhrer A, Fröberg L E, Pedersen J N, Larsson M W, Wacker A, Pistol M and Samuelson L 2007 Few electron double quantum dots in InAs/InP nanowire heterostructures Nano Lett. 7243 [9] Iijima S 1991 Helical microtubules of graphitic carbon Nature 35456

[10] Dai H, Wong E W and Lieber C M 1996 Probing electrical transport in nanomaterials: conductivity of individual carbon nanotubes Science $\mathbf{2 7 2} 523$

[11] Tans S J, Devoret M H, Dai H, Thess A, Smalley R E, Geerligs L J and Dekker C 1997 Individual single-wall carbon nanotubes as quantum wires Nature $\mathbf{3 8 6} 474$ [12] Deshpande V V and Bockrath M 2008 The one-dimensional Wigner crystal in carbon nanotubes Nature 4314

[13] Goni A R, Pfeiffer L N, West K W, Pinczulk A, Baranger H U and Stormer H L 1992 Observation of quantum wire formation at intersecting quantum wells Appl. Phys. Lett. 611956 [14] Yoshita M, Akiyama H, Pfeiffer L N and West K W 2002 Quantum wells with atomically smooth interfaces Appl. Phys. Lett. 8149 
[15] Auslaender O M, Steinberg H, Yacoby A, Tserkovnyak Y, Halperin B I, Baldwin K W, Pfeiffer L N and West K W 2005 Spin-charge separation and localization in one dimension Science $\mathbf{3 0 8} 88$

[16] De Picciotto R, Baldwin K W, Pfieffer L N and West K W 2008 The 0.7 structure in cleaved edge overgrowth wires J. Phys.: Condens. Matter 20164204

[17] Fallahi P 2006 Imaging electrons in few-electron quantum dots PhD thesis, Harvard University

[18] Qian J, Halperin B I and Heller E J 2010 Imaging and manipulating electrons in a onedimensional quantum dot with Coulomb blockade microscopy Phys. Rev. B $\mathbf{8 1} 125323$

[19] Matveev K A 2004 Conductance of a quantum wire at low electron density Phys. Rev. B 70 245319

[20] Boyd E (in press) Imaging electrons in ultra-thin nanowires $\mathrm{PhD}$ thesis, Harvard University [21] Olsson L Ö, Andersson C B M, Håkansson M C, Kanski J, Ilver L and Karlsson U O 1996 Charge accumulation at InAs surfaces Phys. Rev. Lett. 763626

[22] Björk M T, Fuhrer A, Hansen A E, Larsson M W, Fröberg L E and Samuelson L 2005 Tunable effective $g$ factor in InAs nanowire quantum dots Phys. Rev. B 72 201307(R)

[23] Tessmer S H, Glicofridis P I, Ashoori R C, Levitov L S and Melloch M R 1998 Subsurface charge accumulation imaging of a quantum Hall liquid Nature 39251

[24] Topinka M A, LeRoy B J, Shaw S E J, Heller E J, Westervelt R M, Maranowski K D and Gossard A C 2000 Imaging coherent electron flow from a quantum point contact Science $\mathbf{2 8 9}$ 2323

[25] Topinka M A, LeRoy B J, Westervelt R M, Shaw S E J, Fleischmann R, Heller E J and Maranowski K 2001 Coherent branched flow in a two-dimensional electron gas Nature 410183 [26] Fallahi P, Bleszynski A C, Westervelt R M, Huang J, Walls J D, Heller E J, Hanson M and Gossard A C 2005 Imaging a single-electron quantum dot Nano Lett. 223 
[27] Gildemeister A E, Ihn T, Schleser R, Ensslin K, Driscoll D C and Gossard A C 2007 Imaging a coupled quantum dot-quantum point contact system J. Appl. Phys. 102083703 [28] Jura M P, Topinka M A, Urban L, Yazdani A, Shtrikman H, Pfieffer L N, West K W and Goldhaber-Gordon D 2007 Unexpected features of branched flow through high-mobility twodimensional electron gases Nature Phys. 3841

[29] Woodside M T and McEuen P L 2002 Scanned probe imaging of single-electron charge states in nanotube quantum dots Science 2961098

[30] Berezovsky J, Borunda M F, Heller E J and Westervelt R M 2010 Imaging coherent transport in graphene (part I): mapping universal conductance fluctuations Nanotechnology 21 274013

[31] Berezovsky J and Westervelt R M 2010 Imaging coherent transport in graphene (part II): probing weak localization Nanotechnology 21274014

[32] Schnez S, Güttinger J, Huefner M, Stampfer C, Ensslin K and Ihn T 2010 Imaging localized states in graphene nanostructures arXiv:1005.2024v1 [cond-mat.mes-hall] [33] Bleszynski A C, Zwanenburg F A, Westervelt R M, Roest A L, Bakkers E P A M and Kouwenhoven L P 2007 Scanned probe imaging of quantum dots inside InAs nanowires Nano Lett. 72559

[34] Bleszynski-Jayich A C, Fröberg L E, Björk M T, Trodahl H J, Samuelson L and Westervelt R M 2008 Imaging a one-electron InAs quantum dot in an InAs/InP nanowire Phys. Rev. B 77 245327

[35] Jensen L E, Björk M T, Jeppesen S, Persson A I, Ohlsson B J and Samuelson L 2004 Role of surface diffusion in chemical beam epitaxy of InAs nanowires Nano Lett. 41961

[36] Ruzin I M, Chandrasekhar V, Levin E I and Glazman L I 1992 Stochastic Coulomb blockade in a double-dot system Phys. Rev. B 4513469 
[37] Waugh F R, Berry M J, Mar D J, Westervelt R M, Campman K L and Gossard A C 1995

Single-electron charging in double and triple quantum dots with tunable coupling Phys. Rev. Lett. 75705

[38] Yao Z, Dekker C and Avouris P 2001 Electrical transport through single-wall carbon nanotubes Topics in Applied Physics vol 80, ed Dresselhaus M S, Dresselhaus G and Avouris Ph (Berlin: Springer-Verlag) pp 147-171 


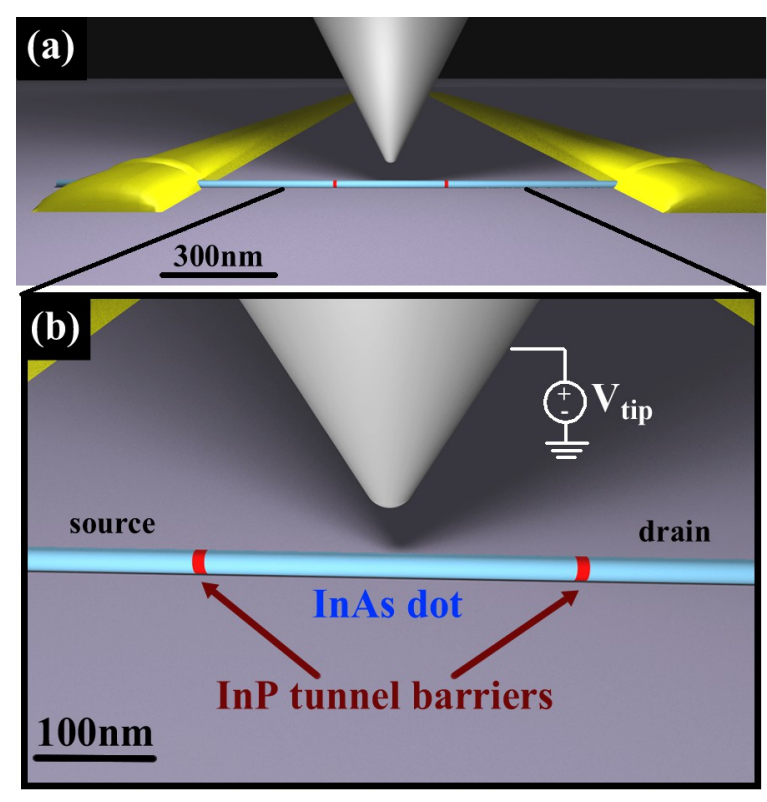

Figure 1. Illustration of our scanning gate microscope (SGM) setup. Two 2-nm-thick InP barriers form a $\sim 300$-nm-long quantum dot in an ultra-thin (30 nm diameter) epitaxially grown InAs nanowire. The nanowire is deposited onto a Si substrate with a capping layer of $100 \mathrm{~nm}$ of thermal oxide and is electrically contacted with $\mathrm{Ni} / \mathrm{Au}$ leads. A backgate (not shown) globally tunes the electron density of the wire. The conducting tip acts as a moveable gate and has a radius of $\sim 20 \mathrm{~nm}$. The applied tip voltage $V_{\text {tip }}$ and the height above the nanowire are independently tuned. The movable gate is held at a constant height and raster scanned. The quantum dot is much longer than it is wide, with a length-to-diameter ratio of $\sim 10$. All features are represented to scale, except the InP barriers, which are enlarged for ease of viewing. 


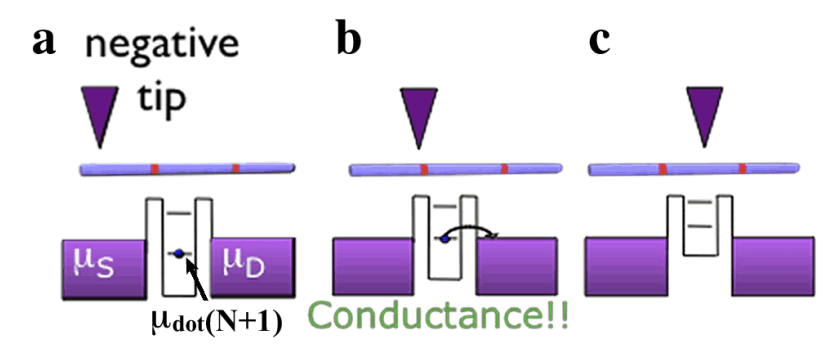

d
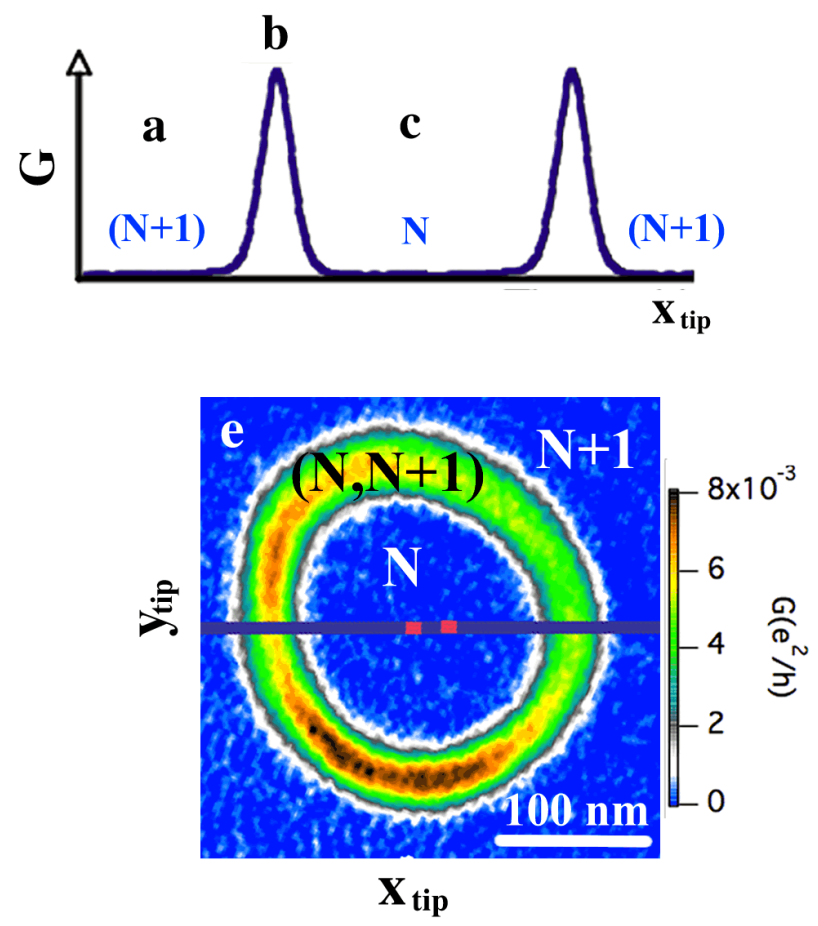

Figure 2: Schematic of the Coulomb blockade imaging technique for zero $V_{s d}$. (a)-(e) Changing the distance between a negatively charged SGM tip and a quantum dot manipulates the charge on the dot by controlling the strength of the tip-to-dot coupling $C_{t-d .}$ (b) Current will only flow through the nanowire when the chemical potential of the dot ${ }_{d o t}$ is in resonance with the source and the drain. In (a) and (c) the negative tip gates the dot so the chemical potential is out of resonance and the current is blocked. (d) A plot of the conductance through the nanowire as the tip is scanned in a straight line along the length of the wire. (e) Translating the plot from a 1D scan to the image obtained when raster scanning over the wire in 2D produces images with Coulomb blockade conductance rings. The nanowire in (e) had a diameter of $50 \mathrm{~nm}$, and the dot length was $18 \mathrm{~nm}$. The blue regions of zero conductance in (d) correspond to locations of the tip where conductance through the wire is blocked. In this manner the charge on the dot is manipulated and the number and location of quantum dots along the nanowire are identified. 

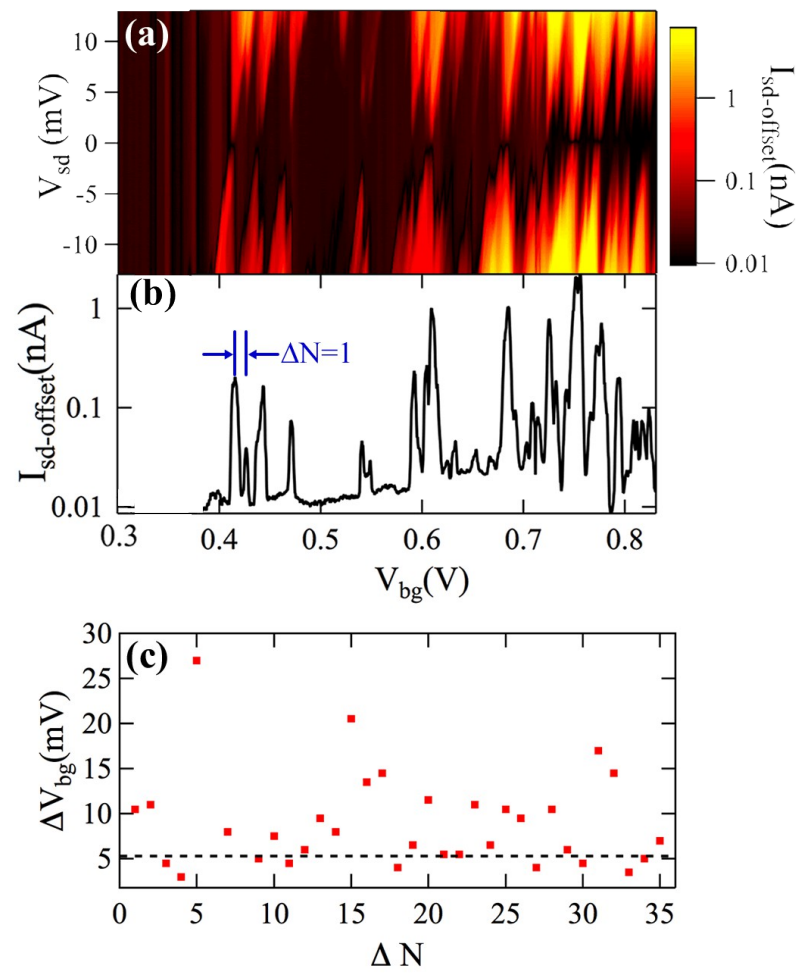

Figure 3. Transport measurements of the ultra-thin nanowire without the tip present at $T=4.2 \mathrm{~K}$. (a) Coulomb blockade plot where offset current $\Gamma_{s d-o f f s e t}=\left\lceil\Gamma_{s d}\lceil+10 \mathrm{pA}\right.$ is plotted $v s$. the backgate voltage $V_{b g}$ and the source-drain bias $V_{s d}$. An offset of $10 \mathrm{pA}$ is added to $\left\lceil\Gamma_{s d}\lceil\right.$ to be able to see the behaviour at low source-drain bias. Complex Coulomb blockade diamonds are seen which would be very difficult to understand without scanning gate measurements. The dots are empty of electrons at zero $V_{b g}$ and a positive backgate voltage must be applied to pull electrons onto the dots. (b) $\left\lceil\Gamma_{s d}\left\lceil\quad v s . V_{b g}\right.\right.$ from figure 3(a) at $V_{s d}=5 \mathrm{mV}$. Coulomb blockade peaks of varying height and spacing are seen. (c) Plot of the measured voltage differences $\Gamma V_{b g}$ between adjacent Coulomb blockade peaks from figure 3(b) vs. the difference $\Gamma \Gamma \Gamma$ in the number of electrons in the quantum dot. The dashed line marks the average $\Gamma V_{b g}=5.5 \mathrm{mV}$ of the closest peak spacings, which corresponds to the voltage difference between peaks due to the longest dot in the system. 

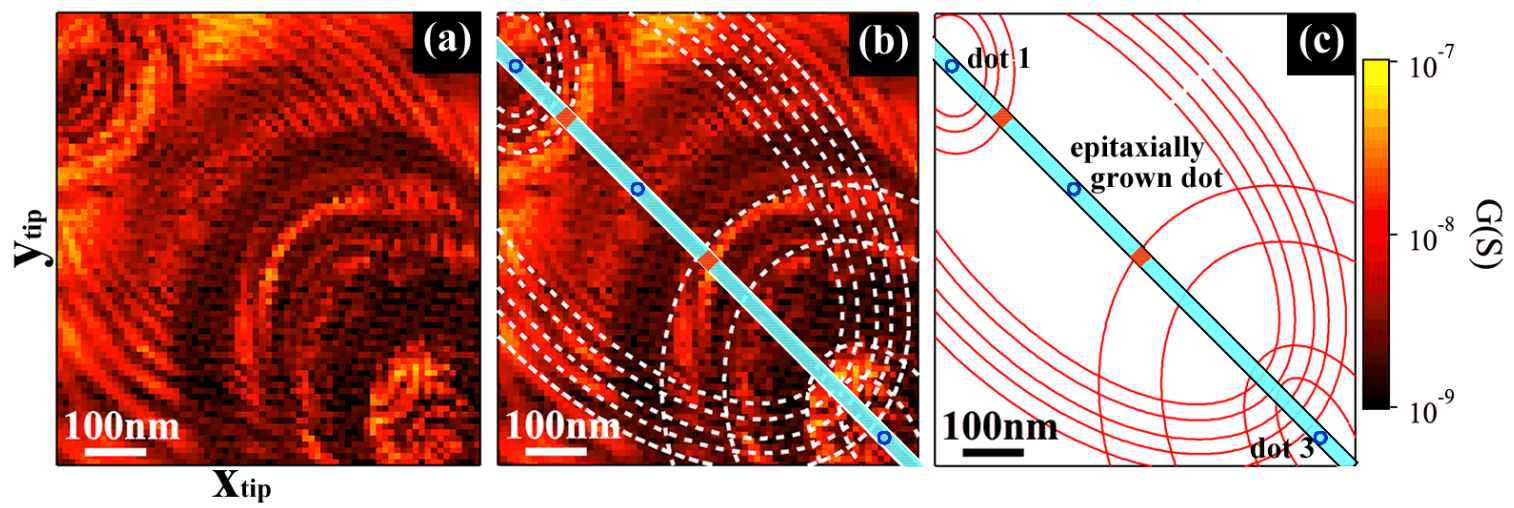

Figure 4. (a) Conductance image of an ultra-thin $\mathrm{InAs} / \mathrm{InP}$ nanowire at $T=4.2 \mathrm{~K}$. The conductance $G$ is plotted on a logarithmic colour scale $v s . x_{t i p}$ and $y_{t i p}$. The tip is scanned in a plane $60 \mathrm{~nm}$ above the nanowire with respect to the $\mathrm{Ni} / \mathrm{Au}$ contacts as shown in figure $5(\mathrm{~g})$. We found three quantum dots in series along the length of the nanowire. The center dot is the epitaxially grown dot, and dots 1 and 3 on either side are likely formed by Schottky barriers at the metal/semiconductor interface. (b) The blue line represents the location of the InAs nanowire and the red regions represent the approximate location of the two InP barriers (barriers are enlarged for ease of viewing). The small blue circles mark the centers of the quantum dots. The dashed white ellipses trace several of the Coulomb blockade conductance rings for each dot. The voltages are $V_{t i p}=-0.7 \mathrm{~V}, V_{s d}=2.5 \mathrm{mV}$, and $V_{b g}=0.724 \mathrm{~V}$. (c) The conductance data is removed and only the nanowire, the InP barriers, the center markers, and the conductance ring fits are sketched.

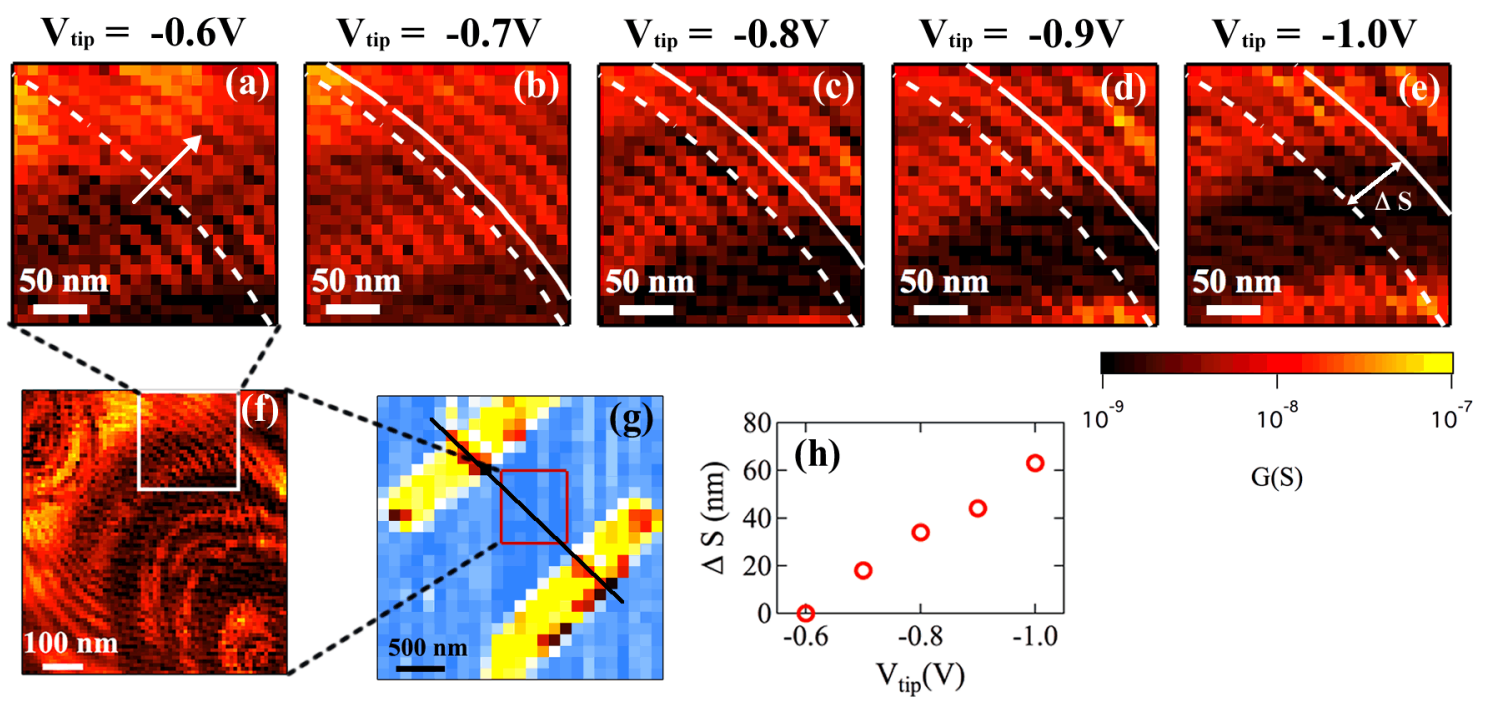


Figure 5. SGM spatial conductance images of an ultra-thin InAs/InP nanowire at $T=4.2 \mathrm{~K}$. A change in the tip voltage or tip position alters the induced charge on the dots: $q=C_{t d}\left(r_{t i p}\right) V_{t i p}$. Figure 5(a)-(e) tracks the spatial progression of a specific conductance ring of the epitaxially grown dot, as the tip voltage is made more negative. The dotted white curve marks the location of the specific conductance ring at $V_{t i p}=-0.6 \mathrm{~V}$. The solid white curves in (b)-(e) track the outward advancement of the same conductance ring as the tip voltage is made more negative. (f) shows the region of interest in (a)-(e). (g) The location of the larger scan area, red box, relative to the Ni/Au leads (yellow) and the nanowire axis (black line). (h) Plot of the outward movement of the conductance ring marked with the dashed white line in figure 5(a) as the tip voltage is made more negative. $\lceil S$ is the separation between the location of the conductance ring at the reference voltage, $V_{\text {tip }}=-0.6 \mathrm{~V}$, and the current value of $V_{t i p}$. A movie of the conductance ring evolution is included with the online supplementary data (file format: AVI, file size: 2.6 MB).
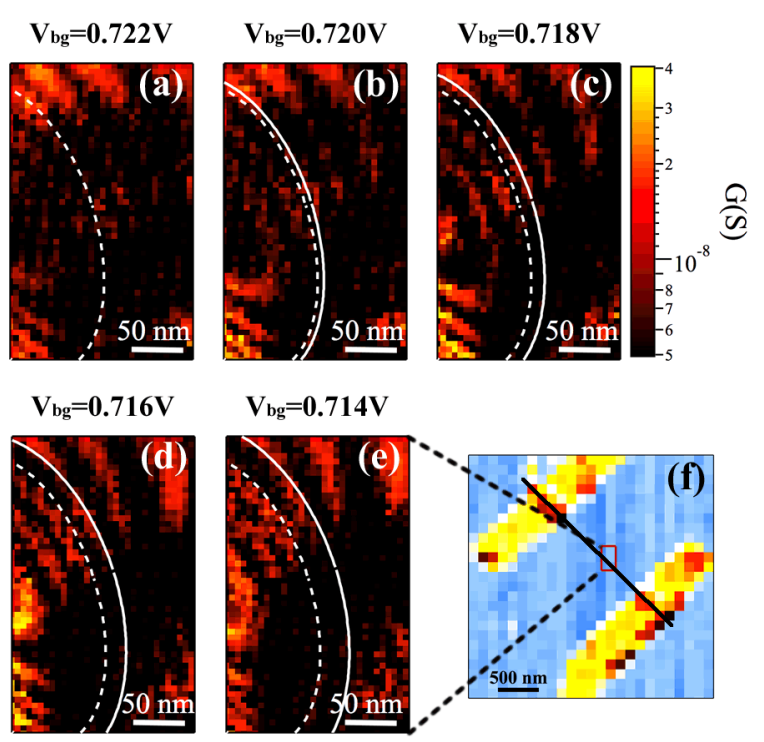

Figure 6. SGM spatial conductance images of an ultra-thin InAs/InP nanowire at $T=4.2 \mathrm{~K}$. Changing the backgate voltage and tip position can also alter the charge state of a quantum dot. Figure 6(a)-(e) tracks the spatial progression of a specific conductance ring of the epitaxially grown dot as the backgate voltage is made more negative. The dotted white curve marks the location of the specific conductance ring at $V_{b g}=0.722 \mathrm{~V}$. The solid white curves in (b)-(e) track the outward advancement of the same conductance ring as the backgate voltage is decreased. (f) The location of the scan area, red box, relative to the $\mathrm{Ni} / \mathrm{Au}$ leads (yellow) and the nanowire axis (black line). 